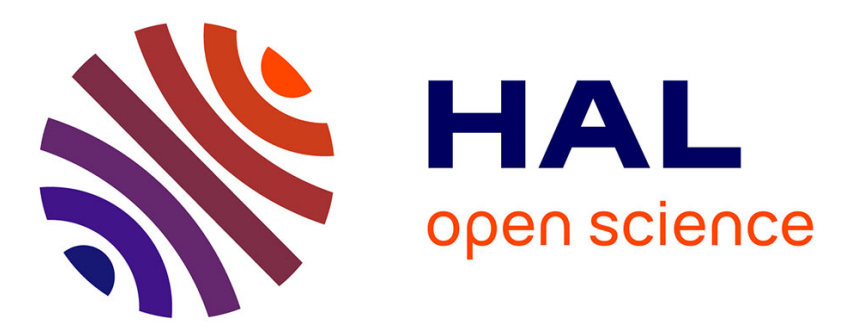

\title{
Discontinuous Galerkin approximation of two-phase flows in heterogeneous porous media with discontinuous capillary pressures
}

\author{
Alexandre Ern, Igor Mozolevski, Luciane Schuh
}

\section{- To cite this version:}

Alexandre Ern, Igor Mozolevski, Luciane Schuh. Discontinuous Galerkin approximation of two-phase flows in heterogeneous porous media with discontinuous capillary pressures. Computer Methods in Applied Mechanics and Engineering, 2010, 199 (23-24), pp.1491-1501. 10.1016/j.cma.2009.12.014 . hal-00368026

\section{HAL Id: hal-00368026 \\ https://hal.science/hal-00368026}

Submitted on 13 Mar 2009

HAL is a multi-disciplinary open access archive for the deposit and dissemination of scientific research documents, whether they are published or not. The documents may come from teaching and research institutions in France or abroad, or from public or private research centers.
L'archive ouverte pluridisciplinaire HAL, est destinée au dépôt et à la diffusion de documents scientifiques de niveau recherche, publiés ou non, émanant des établissements d'enseignement et de recherche français ou étrangers, des laboratoires publics ou privés. 


\title{
Discontinuous Galerkin approximation of two-phase flows in heterogeneous porous media with discontinuous capillary pressures
}

\author{
A. $\operatorname{Ern}^{\mathrm{a}, 1}$, I. Mozolevski ${ }^{\mathrm{a}, \mathrm{b}, 2}$, L. Schuh ${ }^{\mathrm{c}}$ \\ ${ }^{a}$ Université Paris-Est, CERMICS, Ecole des Ponts, $6 \& 8$ Av. B. Pascal, 77455 \\ Marne-la-Vallée cedex 2, France \\ ${ }^{b}$ Departamento de Matemática, Universidade Federal de Santa Catarina, 88040-900, \\ Florianópolis-SC, Brasil \\ ${ }^{c} I M E$, Universidade de São Paulo, Rua do Matão, 1010, Cidade Universitária, \\ 05508-090, São Paulo, SP, Brasil
}

\begin{abstract}
We design and investigate a sequential discontinuous Galerkin method to approximate two-phase immiscible incompressible flows in heterogeneous porous media with discontinuous capillary pressures. The nonlinear interface conditions are enforced weakly through an adequate design of the penalties on interelement jumps of the pressure and the saturation. An accurate reconstruction of the total velocity is considered in the Raviart-Thomas(-Nédélec) finite element spaces, together with diffusivity-dependent weighted averages to cope with degeneracies in the saturation equation and with media heterogeneities. The proposed method is assessed on one-dimensional test cases exhibiting rough solutions, degeneracies, and capillary barriers. Stable and accurate solutions are obtained without limiters.
\end{abstract}

Key words: two-phase flows, heterogeneous porous media, discontinuous capillary pressure, discontinuous Galerkin, interface condition, velocity reconstruction, weighted averages, secondary oil recovery

PACS: 02.70.Dh, 47.56.+r, 02.60.Cb

Email addresses: ern@cermics.enpc.fr (A. Ern), Igor.Mozolevski@mtm.ufsc.br (I. Mozolevski), lucianes@ime.usp.br (L. Schuh)

${ }^{1}$ Partially supported by the Groupement MoMaS (PACEN/CNRS, ANDRA, BRGM, CEA, EdF, IRSN), France

${ }^{2}$ Partially supported by CNPq, Brazil 
2000 MSC: 65M60, 65N30, 76T99, 76S05

\section{Introduction}

Two-phase immiscible incompressible flows through porous media are important in many applications, e.g., secondary oil recovery in petroleum engineering [2]. Swift changes of rock properties in an oil reservoir lead to capillary pressure discontinuities which can considerably reduce the recovery factor and even cause oil trapping; see, e.g., [7, 23, 27]. For such applications, the development of numerical methods that can cope satisfactorily with two-phase flows in heterogeneous porous media with discontinuous capillary pressures is a relevant and challenging problem.

The physical principles governing two-phase immiscible incompressible flows in porous media and the corresponding mathematical models can be found, e.g., in $[6,13,15,25]$. The governing equations generally consist of an elliptic Darcy-type equation for the pressure and a nonlinear degenerate parabolic equation with a nonlinear advective term for the saturation. The dependent variables are either the total or the wetting-phase pressure and either the wetting or the non-wetting phase saturation. The governing equations are often solved sequentially: at each time step, the pressure equation is solved first, then the total velocity is computed from the pressure gradient, and finally, the saturation equation is advanced in time using the total velocity in the advective term. This approach substantially alleviates the nonlinearities in the coupled system of governing equations. Furthermore, working with the global pressure instead of the wetting-phase pressure offers the advantage of less strongly coupled equations, since the capillary pressure no longer enters explicitly the pressure equation.

Discontinuous Galerkin methods (DG) offer an attractive tool to approximate (in space) two-phase flows in porous media. Advantages of such methods include their local (elementwise) conservation property, their flexibility in using non-matching meshes and variable polynomial degrees, and their potential to capture shocks sharply while maintaining high accuracy in smooth regions. A mathematical analysis of DG methods applied to Friedrichs' systems encompassing both elliptic and hyperbolic PDE's can be found in [19]. Different versions of DG methods have been considered for the sequential pressure-saturation formulation of two-phase flows. In [5], the nonsymmetric interior penalty $\mathrm{DG}$ method was applied to the pressure equation and to 
the diffusive term in the saturation equation, upwinding was used for the advective term, and the total velocity was recovered using the postprocessing proposed in [4]. Similar techniques together with further variants of DG methods for the diffusive terms were investigated numerically in [26] in the context of adaptive methods. In [24], a compressible air-water two-phase problem was studied numerically using various variants of DG methods for the pressure equation and the so-called Local DG method for the saturation equation. In general, when approximating rough solutions by DG methods, spurious oscillations triggered by Gibbs-type phenomena occur and, in the context of two-phase flows, can become significant. To control them, slope limiters can be used, as advocated for instance in [5]. However, slope limiters are fairly complicated to be designed for unstructured meshes and there is no analysis available in two or three space dimensions. One possibility proposed and assessed numerically in [18] to avoid spurious oscillations is to consider a fully implicit DG method for the complete coupled system of pressure and saturation equations, but this entails substantial computational costs.

All of the above DG methods for two-phase flows cannot cope satisfactorily with capillary pressure discontinuities produced at the interface between two subdomains with different rock properties. Indeed, such discontinuities require a nonlinear interface condition for the pressure and the saturation, and both variables can exhibit a nonzero jump at the interface; see $[6,7,17]$ for a detailed discussion. As a result, the usual paradigm in the penalty strategy of DG methods must be revisited. Formulating a suitable penalty strategy to incorporate weakly the nonlinear interface conditions on the pressure and on the saturation in the context of sequential pressure-saturation formulations constitutes the first novel contribution of this work. From a mathematical viewpoint, existence and uniqueness of the weak solution to the coupled system of pressure-saturation equations with general interface conditions was analyzed in $[1,8]$ using vanishing viscosity and homogenization techniques. Moreover, in the past few years, two-phase flows with capillary pressure discontinuities have been studied extensively in the context of Finite Volume methods (FV). An interface problem for a nonlinear degenerate parabolic equation, representative of the saturation equation with discontinuous capillary pressure, was analyzed in [17]. A FV scheme for the approximation of the solution was proposed and shown to converge to a weak solution. A more general formulation of the interface conditions was considered in $[10,12]$, where a new definition of a weak solution for such interface conditions was introduced; existence and uniqueness of the weak solution 
was proven. Interface problems for degenerate parabolic equations including a nonlinear advective term with a discontinuity at the interface for the flux function were analyzed in [11]. The above FV schemes solely handle the saturation equation with a prescribed total velocity field, whereas the present work will address the coupled system of pressure-saturation equations where the total velocity is also an unknown.

The second contribution of the present work is to propose some possible improvements in the implementation of DG methods for two-phase flows in heterogeneous porous media. These improvements concern the total velocity reconstruction and the use of weighted averages in the formulation of the DG method. Firstly, we consider the total velocity reconstruction introduced in [21] for elliptic problems and extended to two-phase flow problems in [20]. This velocity reconstruction offers various advantages with respect to the reconstruction proposed in [4]. While both reconstructions enforce continuity of the normal component of the total velocity at mesh interfaces and hence produce a total velocity field in the space $\mathbf{H}(\mathrm{div})$ defined below, the present reconstruction exploits all the degrees of freedom in the DG method and, as such, produces an optimal approximation of the divergence of the total velocity. As a result, it leads to more accurate and stable calculations, as illustrated numerically in [20]. A further advantage is that even for piecewise affine discrete pressures, the reconstructed velocity already belongs to the first-order Raviart-Thomas(-Nédélec) finite element space defined below. Therefore, it is possible to consider the natural setting of equal-order interpolation (e.g., piecewise affine) for the pressure and the saturation. In the present work, we extend this reconstruction to the more complex situation where the pressure and the saturation can exhibit nonzero jumps at an interface owing to capillary pressure discontinuity. Secondly, following previous work on DG methods for advection-diffusion equations in heterogeneous media [16, 22], we formulate the consistency terms in the DG method using weighted averages instead of the more usual arithmetic averages, thereby allowing to use the harmonic average of the corresponding diffusivity to penalize interelement jumps. Weighted averages are used in both pressure and saturation equations, and are particularly important in the latter because of possible degeneracies.

The paper is organized as follows. In $\S 2$, we briefly restate the governing equations for two-phase flows in porous media in the coupled total pressure/fractional flow formulation and present the interface conditions induced by capillary pressure discontinuities. In $\S 3$, we present the sequential DG dis- 
cretization of the governing equations with weighted averages, the penalty strategy to incorporate the interface conditions, and the accurate total velocity reconstruction. Finally, $\S 4$ contains the numerical results. No limiters are used in this work.

\section{Problem formulation}

\subsection{The setting}

Let $\Omega$ be a bounded, open, polyhedral domain in $\mathbb{R}^{d}, d \geq 1$, with boundary $\partial \Omega$ and outward normal $n_{\partial \Omega}$. The domain $\Omega$ is divided into two open, polyhedral subdomains $\Omega_{1}$ and $\Omega_{2}$ such that $\bar{\Omega}=\overline{\Omega_{1}} \cup \overline{\Omega_{2}}$ and $\Omega_{1} \cap \Omega_{2}=\emptyset$. Let $\Gamma:=\partial \Omega_{1} \cap \partial \Omega_{2}$ denote the interface between the two subdomains. More general situations with more than two subdomains can also be considered.

Each subdomain $\Omega_{\beta}, \beta \in\{1,2\}$, represents a porous medium with porosity $\phi_{\beta}$ and intrinsic (absolute) permeability $K_{\beta}$; both quantities are assumed to be constant in each subdomain for simplicity. The mobility of the wetting $(\alpha=w)$ and of the non-wetting phase $(\alpha=n)$ in the subdomain $\Omega_{\beta}$ is denoted by $\lambda_{\alpha \beta}$, the sum $\lambda_{\beta}:=\lambda_{w \beta}+\lambda_{n \beta}$ being the total mobility and $f_{\beta}:=\frac{\lambda_{n \beta}}{\lambda_{\beta}}$ the fractional flux. Let $\pi_{\beta}$ be the capillary pressure in the subdomain $\Omega_{\beta}$. In the sequel, the index $\beta$ is used to indicate the restriction of a function defined on $\Omega$ to the subdomain $\Omega_{\beta}$.

The capillary pressure and the mobilities are in each subdomain given smooth functions of the non-wetting phase saturation. The non-wetting phase saturation takes values in the interval $\left[S_{n r}, 1-S_{w r}\right]$, and up to rescaling, we can assume that the same interval is used in both subdomains. Here, $S_{n r}$ and $S_{w r}$ respectively denote the residual saturation of the wetting and non-wetting phase. In each subdomain $\Omega_{\beta}, \beta \in\{1,2\}$, the capillary pressure $\pi_{\beta}:\left[S_{n r}, 1-S_{w r}\right) \rightarrow[0,+\infty)$ is an increasing, continuously differentiable function. We assume that

$$
\forall s \in\left[S_{n r}, 1-S_{w r}\right), \quad \pi_{1}(s)<\pi_{2}(s) .
$$

Moreover, we assume that $\pi_{2}\left(S_{n r}\right)<\lim _{s \rightarrow 1-S_{w r}} \pi_{1}(s)$. As a result, there is a critical value $S^{*}$ of the non-wetting phase saturation such that

$$
\pi_{1}\left(S^{*}\right)=\pi_{2}\left(S_{n r}\right)
$$

This situation is illustrated in Figure 1 . The value $\pi_{\beta}\left(S_{n r}\right)$ is called an entry pressure and corresponds to the capillary pressure needed to displace the 


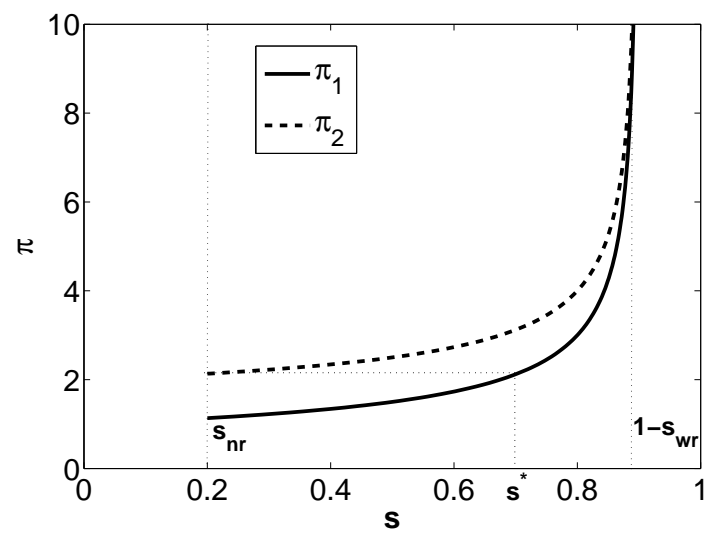

Figure 1: Capillary pressures in subdomains $\Omega_{1}$ and $\Omega_{2}$ and critical non-wetting phase saturation $s^{*}$.

non-wetting phase from the largest pore in the subdomain $\Omega_{\beta}$. The difference between entry pressures in $\Omega_{1}$ and $\Omega_{2}$ is at the origin of the oil trapping effect [7]. Finally, regarding the mobilities, we assume that $\lambda_{n \beta}:\left[S_{n r}, 1-S_{w r}\right] \rightarrow$ $[0,+\infty)$ and $\lambda_{w \beta}:\left[S_{n r}, 1-S_{w r}\right] \rightarrow[0,+\infty)$ are monotone Lipschitz functions such that $\lambda_{n \beta}\left(S_{n r}\right)=0$ and $\lambda_{w \beta}\left(1-S_{w r}\right)=0$. We also assume that the fractional fluxes $f_{\beta}:\left[S_{n r}, 1-S_{w r}\right] \rightarrow[0,1]$ are increasing functions. Usually, these functions have a so-called $S$-shape.

\subsection{Governing equations}

The governing equations for two-phase immiscible incompressible flows through the heterogeneous porous medium $\Omega$ in the global pressure/fractional flow formulation and in the absence of gravity forces can be written as follows: For a given simulation time $\mathfrak{T}>0$, find $(p, s)$ that satisfy in $\Omega_{\beta} \times[0, \mathfrak{T}]$ for each $\beta \in\{1,2\}$,

$$
\begin{aligned}
& -\nabla \cdot\left(\lambda_{\beta}\left(s_{\beta}\right) K_{\beta} \nabla p_{\beta}\right)=q_{w \beta}+q_{n \beta}, \\
& u_{\beta}=-\lambda_{\beta}\left(s_{\beta}\right) K_{\beta} \nabla p_{\beta}, \\
& \phi_{\beta} \partial_{t} s_{\beta}+\nabla \cdot\left(u_{\beta} f_{\beta}\left(s_{\beta}\right)\right)-\nabla \cdot\left(\epsilon_{\beta}\left(s_{\beta}\right) \nabla \pi_{\beta}\left(s_{\beta}\right)\right)=q_{n \beta},
\end{aligned}
$$

where the dependent variables $p$ and $s$ respectively denote the global pressure (in short, the pressure) and the non-wetting phase saturation (in short, the saturation), while $u$ denotes the total velocity. In addition, we have set 
$\epsilon_{\beta}:=\lambda_{w \beta} f_{\beta} K_{\beta}$, and $q_{\alpha}, \alpha \in\{w, n\}$, denotes volumetric sources or sinks of the corresponding phase in the medium. Observe that the pressure equation does not degenerate, whereas the saturation equation does in the limits $s \rightarrow S_{n r}$ and $s \rightarrow 1-S_{w r}$.

Interface conditions on $\Gamma$ are discussed in the following subsection. The initial condition prescribes the saturation over the subdomains $\Omega_{\beta}$ at initial time. We assume that the wetting phase is initially present in the whole domain. Boundary conditions can be of Dirichlet or Neumann type for both the pressure and the saturation. Dirichlet boundary conditions are enforced on the sets $\partial \Omega_{p}^{\mathrm{D}}$ and $\partial \Omega_{s}^{\mathrm{D}}$ for the pressure and the saturation, respectively, both sets being assumed to have nonzero $(d-1)$-dimensional measure. In addition, $\partial \Omega_{s}^{\mathrm{D}}$ coincides with the inflow boundary defined such that $u \cdot n_{\partial \Omega}<0$. Neumann boundary conditions enforce the normal component of the total velocity, namely $\lambda(s) K \nabla p \cdot n_{\partial \Omega}$, or the normal component of the saturation diffusive flux, namely $\epsilon(s) \nabla \pi(s) \cdot n_{\partial \Omega}$. For simplicity, we only consider homogeneous Neumann boundary conditions. For a further discussion of practically important boundary conditions in the global pressure/fractional flow formulation, we refer to $[13,14]$.

\subsection{Interface conditions}

We follow here the formulation of interface conditions in $[13,17]$. The interface conditions for the saturation enforce the continuity of the normal component of the total advective-diffusive flux, prescribe the value of $s_{2}$ to $S_{n r}$ if $s_{1} \in\left[S_{n r}, S^{*}\right]$, and enforce the continuity of the capillary pressure if $s_{1} \in\left[S^{*}, 1-S_{w r}\right)$. This yields on $\Gamma$,

$$
\left(u_{1} f_{1}\left(s_{1}\right)-\epsilon_{1}\left(s_{1}\right) \nabla \pi_{1}\left(s_{1}\right)\right) \cdot n_{\Gamma}=\left(u_{2} f_{2}\left(s_{2}\right)-\epsilon_{2}\left(s_{2}\right) \nabla \pi_{2}\left(s_{2}\right)\right) \cdot n_{\Gamma},
$$

where $n_{\Gamma}$ is a unit normal vector to $\Gamma$, along with

$$
\begin{aligned}
s_{2} & =S_{n r} & & \text { if } s_{1} \in\left[S_{n r}, S^{*}\right], \\
\pi_{1}\left(s_{1}\right) & =\pi_{2}\left(s_{2}\right) & & \text { if } s_{1} \in\left[S^{*}, 1-S_{w r}\right) .
\end{aligned}
$$

Introducing the function $J:\left[S_{n r}, 1-S_{w r}\right) \rightarrow[0,+\infty)$ such that

$$
J(s)= \begin{cases}s & \text { if } s_{1} \in\left[S_{n r}, S^{*}\right], \\ s-\pi_{2}^{-1}\left(\pi_{1}(s)\right) & \text { if } s_{1} \in\left[S^{*}, 1-S_{w r}\right),\end{cases}
$$


where $\pi_{2}^{-1}$ denotes the reciprocal function of $\pi_{2}$, the interface conditions (7)(8) yield

$$
s_{1}-s_{2}=J\left(s_{1}\right) .
$$

Observe that $J$ is continuous at $S^{*}$.

The interface conditions for the pressure enforce the continuity of the normal component of the total velocity and prescribe the continuity of the wetting phase pressures $p_{w \beta}$ if $s_{1} \in\left[S_{n r}, S^{*}\right]$ and that of the non-wetting phase pressures $p_{n \beta}$ if $s_{1} \in\left[S^{*}, 1-S_{w r}\right)$. In the latter case, continuity of the capillary pressures holds owing to (8), thereby implying also the continuity of the wetting phase pressures. Thus, the wetting-phase pressure is always continuous, in agreement with the fact that this phase is always present in both subdomains. Altogether, this yields on $\Gamma$,

$$
-\lambda_{1} K_{1} \nabla p_{1} \cdot n_{\Gamma}=-\lambda_{2} K_{2} \nabla p_{2} \cdot n_{\Gamma}
$$

along with

$$
\begin{array}{ll}
p_{1}-p_{2}=g\left(s_{1}\right) & \text { if } s_{1} \in\left[S_{n r}, S^{*}\right], \\
p_{1}-p_{2}=\hat{g}\left(s_{1}, s_{2}\right) & \text { if } s_{1} \in\left[S^{*}, 1-S_{w r}\right),
\end{array}
$$

where we have introduced the functions

$$
\begin{aligned}
g\left(s_{1}\right) & :=\int_{S_{n r}}^{s_{1}} f_{1}(\xi) \pi_{1}^{\prime}(\xi) d \xi+\pi_{1}\left(S_{n r}\right)-\pi_{2}\left(S_{n r}\right), \\
\hat{g}\left(s_{1}, s_{2}\right) & :=\int_{S_{n r}}^{s_{1}}\left(f_{1}(\xi)-1\right) \pi_{1}^{\prime}(\xi) d \xi-\int_{S_{n r}}^{s_{2}}\left(f_{2}(\xi)-1\right) \pi_{2}^{\prime}(\xi) d \xi .
\end{aligned}
$$

Conditions (12) and (13) can be readily linked to the continuity of the wetting and non-wetting phase pressures, respectively, using the definition of the global pressure

$$
p_{\beta}=p_{w \beta}+\int_{S_{n r}}^{s} f_{\beta}(\xi) \pi_{\beta}^{\prime}(\xi) d \xi+\pi_{\beta}\left(S_{n r}\right)=p_{n \beta}+\int_{S_{n r}}^{s}\left(f_{\beta}(\xi)-1\right) \pi_{\beta}^{\prime}(\xi) d \xi
$$

and the fact that $\pi_{\beta}=p_{n \beta}-p_{w \beta}$. Finally, observe that the pressure jump $\left(p_{1}-p_{2}\right)$ is a continuous function of $s_{1}$ since $g\left(S^{*}\right)=\hat{g}\left(S^{*}, S_{n r}\right)$. 


\section{The sequential DG method with total velocity reconstruction}

\subsection{The discrete setting}

Let $\left\{t^{n}\right\}_{0 \leq n \leq N}$ be a sequence of discrete times with $t^{0}=0$ and $t^{N}=\mathfrak{T}$ and time steps $\tau_{n}:=t^{n+1}-t^{n}$. Let $\left\{\mathcal{T}_{h}\right\}_{h>0}$ be a family of shape-regular meshes of the domain $\Omega$. For simplicity, meshes are kept fixed in time. A generic element of $\mathcal{T}_{h}$ is denoted by $T, h_{T}$ stands for its diameter, and $h:=\max _{T \in \mathcal{T}_{h}} h_{T}$ represents the mesh size. We say that $F$ is an interior face of the mesh if $F$ has nonzero $(d-1)$-dimensional measure and if there are distinct $T^{-}$and $T^{+}$in $\mathcal{T}_{h}$ such that $F=\partial T^{-} \cap \partial T^{+}$. We say that $F$ is a boundary face if $F$ has nonzero $(d-1)$-dimensional measure and if there is $T \in \mathcal{T}_{h}$ such that $F=\partial T \cap \partial \Omega$. Interior faces are collected in the set $\mathcal{F}_{h}^{i}$ and boundary faces are collected in the set $\mathcal{F}_{h}^{\partial}$. We also set $\mathcal{F}_{h}:=\mathcal{F}_{h}^{i} \cup \mathcal{F}_{h}^{\partial}$, and for $F \in \mathcal{F}_{h}, h_{F}$ denotes the diameter of $F$. Meshes can possess so-called hanging nodes, resulting for instance from the fact that the subdomains $\Omega_{1}$ and $\Omega_{2}$ have been meshed independently; in this case, we make the usual assumption that there is $\kappa>0$ independent of $h$ such that $h_{F} \geq \kappa h_{T}$ for all $F \in \mathcal{F}_{h}$ and for all $T \in \mathcal{T}_{h}$ such that $F \subset \partial T$.

We assume that the meshes are fitted to the partition of $\Omega$ into $\Omega_{1} \cup \Omega_{2}$. In other words, we assume that the interface $\Gamma$ is exactly covered by a set of faces that is denoted by $\mathcal{F}_{h}^{\Gamma}$. For further use, we define $\mathcal{F}_{h}^{i *}:=\mathcal{F}_{h}^{i} \backslash \mathcal{F}_{h}^{\Gamma}$. We also assume that the sets $\partial \Omega_{p}^{\mathrm{D}}$ and $\partial \Omega_{s}^{\mathrm{D}}$ where Dirichlet boundary conditions are enforced on the pressure and on the saturation, respectively, are exactly covered by boundary faces, and we denote by $\mathcal{F}_{h p}^{\mathrm{D}}$ and $\mathcal{F}_{h s}^{\mathrm{D}}$ the corresponding sets of faces. Since the sets $\Gamma, \partial \Omega_{p}^{\mathrm{D}}$, and $\partial \Omega_{s}^{\mathrm{D}}$ are known a priori, the above are mild assumptions on the meshes.

For a scalar- or vector-valued function $v$ that is possibly two-valued at $F \in \mathcal{F}_{h}^{i}$, we define its jump and average at $F$ as

$$
\llbracket v \rrbracket:=v^{-}-v^{+}, \quad\{v\}:=\frac{1}{2}\left(v^{-}+v^{+}\right), \quad v^{ \pm}:=\left.v\right|_{T^{ \pm}} .
$$

These definitions are extended to boundary faces $F \in \mathcal{F}_{h}^{\partial}$ by setting

$$
\llbracket v \rrbracket=\{v\}:=\left.v\right|_{F} .
$$

For $F \in \mathcal{F}_{h}^{i}$, we define $n_{F}$ as the unit normal vector to $F$ pointing from $T^{-}$ toward $T^{+}$, whereas for $F \in \mathcal{F}_{h}^{\partial}, n_{F}$ coincides with $n_{\partial \Omega}$. The arbitrariness in the orientation of $n_{F}$ is irrelevant in the sequel. For $F \in \mathcal{F}_{h}^{\Gamma}$, it is convenient to choose $n_{F}$ pointing from $\Omega_{1}$ toward $\Omega_{2}$ so that $\llbracket v \rrbracket=v_{1}-v_{2}$ (recall that $\left.v_{\beta}:=\left.v\right|_{\Omega_{\beta}}, \beta \in\{1,2\}\right)$. 


\subsection{Weighted averages}

In the sequel, we consider weighted averages prompted by heterogeneous diffusivity $[16,22]$. Let $a$ be a scalar-valued function representing the diffusivity; $a$ can be two-valued on $F \in \mathcal{F}_{h}^{i}$ with values $a_{T^{-}, F}$ and $a_{T^{+}, F}$ associated with $T^{-}$and $T^{+}$, respectively, whereas $a$ is single-valued on $F \in \mathcal{F}_{h}^{\partial}$. Then, for all $F \in \mathcal{F}_{h}^{i}$, introducing the weights

$$
\omega_{T^{-}, F}:=\frac{a_{T^{+}, F}}{a_{T^{-}, F}+a_{T^{+}, F}}, \quad \omega_{T^{+}, F}:=\frac{a_{T^{-}, F}}{a_{T^{-}, F}+a_{T^{+}, F}},
$$

such that $\omega_{T^{-}, F}+\omega_{T^{+}, F}=1$, the weighted average of a function $v$ is defined as

$$
\{v\}_{\omega}:=\omega_{T^{-}, F} v^{-}+\omega_{T^{+}, F} v^{+} .
$$

We also denote the harmonic mean of $a$ at $F$ as

$$
\langle a\rangle_{F}:=\frac{a_{T^{-}, F} a_{T^{+}, F}}{a_{T^{-}, F}+a_{T^{+}, F}} .
$$

The above definitions are extended to boundary faces by setting $\{v\}_{\omega}:=v$ and $\langle a\rangle_{F}:=a$. Thus, the definitions (19) and (21) can still be used for $F \in \mathcal{F}_{h}^{\partial}$ upon formally setting $a_{T^{-}, F}:=a$ and $a_{T^{+}, F}:=+\infty$. When working with possibly degenerate problems, such as two-phase flow problems, the diffusivity $a$ takes nonnegative (and not necessarily positive) values. The definitions (19) and (21) can still be used if only one of the quantities $a_{T^{ \pm}, F}$ vanishes. If both quantities vanish, the weights are set to $\frac{1}{2}$; for further use, the harmonic mean is set to a global reference value for $a$ specified below.

\subsection{The sequential $D G$ scheme}

Let $k \geq 1$ be an integer. At each discrete time $t^{n}$ with $n \in\{1, \ldots, N\}$, approximations to the pressure, $p_{h}^{n}$, and to the saturation, $s_{h}^{n}$, are sought in the DG finite element space

$$
V_{h}^{k}:=\left\{v_{h} \in L^{2}(\Omega) ; \forall T \in \mathcal{T}_{h},\left.v_{h}\right|_{T} \in \mathbb{P}_{k}(T)\right\},
$$

where $\mathbb{P}_{k}(T)$ denotes the vector space of polynomials with total degree $\leq k$ on $T$. Observe that approximations for the pressure and the saturation are sought using the same polynomial order. Taking different polynomial orders is also possible, but the present choice appears to be more natural. At the initial time, an approximate saturation $s_{h}^{0}$ is constructed by considering, for instance, the $L^{2}$-orthogonal projection onto $V_{h}^{k}$ of the initial datum for the saturation. 


\subsubsection{Step 1: pressure equation}

For $n \in\{0, \cdots, N-1\}$, with $s_{h}^{n} \in V_{h}^{k}$ given from the previous time step $(n \geq 1)$ or by the initial datum $(n=0)$, we first solve the pressure equation using an interior penalty DG method, that is, we solve for $p_{h}^{n+1} \in V_{h}^{k}$ such that for all $z_{h} \in V_{h}^{k}$,

$$
\begin{aligned}
& \sum_{T \in \mathcal{T}_{h}} \int_{T} \lambda\left(s_{h}^{n}\right) K \nabla p_{h}^{n+1} \cdot \nabla z_{h} \\
& -\sum_{F \in \mathcal{F}_{h}^{i} \cup \mathcal{F}_{h p}^{\mathrm{D}}} \int_{F}\left(n_{F} \cdot\left\{\lambda\left(s_{h}^{n}\right) K \nabla p_{h}^{n+1}\right\}_{\omega} \llbracket z_{h} \rrbracket+\theta n_{F} \cdot\left\{\lambda\left(s_{h}^{n}\right) K \nabla z_{h}\right\}_{\omega} \llbracket p_{h}^{n+1} \rrbracket\right) \\
& +\sum_{F \in \mathcal{F}_{h}^{i} \cup \mathcal{F}_{h p}^{\mathrm{D}}} \gamma_{F} \frac{\sigma_{F}}{h_{F}} \llbracket p_{h}^{n+1} \rrbracket \llbracket z_{h} \rrbracket=\sum_{T \in \mathcal{T}_{h}} \int_{T}\left(q_{w}+q_{n}\right) z_{h} \\
& +\sum_{F \in \mathcal{F}_{h}^{\Gamma}} \int_{F}\left(-\theta n_{F} \cdot\left\{\lambda\left(s_{h}^{n}\right) K \nabla z_{h}\right\}_{\omega}+\gamma_{F} \frac{\sigma_{F}}{h_{F}} \llbracket z_{h} \rrbracket\right) G\left(s_{h}^{n}\right) \\
& +\sum_{F \in \mathcal{F}_{h p}^{\mathrm{D}}} \int_{F}\left(-\theta n_{F} \cdot \lambda\left(s_{h}^{n}\right) K \nabla z_{h}+\gamma_{F} \frac{\sigma_{F}}{h_{F}} z_{h}\right) p_{D}
\end{aligned}
$$

where $p_{D}$ is the Dirichlet datum for the pressure. Notice that (23) is a linear problem for $p_{h}^{n+1}$. The interface operator $G\left(s_{h}^{n}\right)$ is defined as

$$
G(s)= \begin{cases}g\left(s_{h 1}^{n}\right), & \text { if } s_{h 1}^{n} \in\left[S_{n r}, S^{*}\right], \\ \hat{g}\left(s_{h 1}^{n}, s_{h 2}^{n}\right), & \text { if } s_{h 1}^{n} \in\left[S^{*}, 1-S_{w r}\right),\end{cases}
$$

where $s_{h \beta}^{n}, \beta \in\{1,2\}$, denotes the restriction of $s_{h}^{n}$ to the subdomain $\Omega_{\beta}$. The parameter $\theta \in\{-1,0,+1\}$ allows to select the so-called nonsymmetric, incomplete, or symmetric version of the interior penalty DG method, and $\sigma_{F}>0$ is a positive parameter that must be taken larger than a minimal threshold depending on mesh regularity if $\theta \neq-1$. This dependency on mesh regularity can be avoided by penalizing liftings of jumps instead of jumps as detailed in $[3,16]$.

The penalty coefficient $\gamma_{F}$ and the weights $\omega$ in the consistency terms are evaluated as described in $\S 3.2$. For $F \in \mathcal{F}_{h}^{i}, F=\partial T^{-} \cap \partial T^{+}$, we set $a_{T^{ \pm}, F}=\left\|\left.\left(\lambda\left(s_{h}^{n}\right) K\right)\right|_{T^{ \pm}}\right\|_{L^{\infty}(F)}$ and for $F \in \mathcal{F}_{h}^{\partial}, F=\partial T \cap \partial \Omega$, we set $a=$ $\left\|\left.\left(\lambda\left(s_{h}^{n}\right) K\right)\right|_{T}\right\|_{L^{\infty}(F)}$. Then, for $F \in \mathcal{F}_{h}^{i *} \cup \mathcal{F}_{h p}^{\mathrm{D}}$, we set

$$
\gamma_{F}=\langle a\rangle_{F}
$$


Recall that the pressure equation does not degenerate, so that the sole motivation for using weighted averages in this equation is media heterogeneities.

It is interesting to recast (23) in a weak residual form by integrating by parts the first term. Observing that for all $F \in \mathcal{F}_{h}^{i}$,

$$
\llbracket \lambda\left(s_{h}^{n}\right) K \nabla p_{h}^{n+1} z_{h} \rrbracket=\left\{\lambda\left(s_{h}^{n}\right) K \nabla p_{h}^{n+1}\right\}_{\omega} \llbracket z_{h} \rrbracket+\llbracket \lambda\left(s_{h}^{n}\right) K \nabla p_{h}^{n+1} \rrbracket\left\{z_{h}\right\}_{\bar{\omega}},
$$

with $\left\{z_{h}\right\}_{\bar{\omega}}:=\left.\omega_{T^{+}, F} z_{h}\right|_{T^{-}}+\left.\omega_{T^{-}, F} z_{h}\right|_{T^{+}}$, and rearranging terms yields

$$
\begin{aligned}
& \sum_{T \in \mathcal{T}_{h}} \int_{T}-\left(\nabla \cdot\left(\lambda\left(s_{h}^{n}\right) K \nabla p_{h}^{n+1}\right)+q_{w}+q_{n}\right) z_{h} \\
& +\sum_{F \in \mathcal{F}_{h}^{i}} \int_{F} n_{F} \cdot \llbracket \lambda\left(s_{h}^{n}\right) K \nabla p_{h}^{n+1} \rrbracket\left\{z_{h}\right\}_{\bar{\omega}}+\sum_{F \in \mathcal{F}_{h}^{\partial} \backslash \mathcal{F}_{h p}^{\mathrm{D}}} \int_{F} n_{F} \cdot \lambda\left(s_{h}^{n}\right) K \nabla p_{h}^{n+1} z_{h} \\
& +\sum_{F \in \mathcal{F}_{h}^{i} \cup \mathcal{F}_{h p}^{\mathrm{D}}} \int_{F}\left(-\theta n_{F} \cdot\left\{\lambda\left(s_{h}^{n}\right) K \nabla z_{h}\right\}_{\omega}+\gamma_{F} \frac{\sigma_{F}}{h_{F}} \llbracket z_{h} \rrbracket\right) \llbracket p_{h}^{n+1} \rrbracket^{\prime}=0,
\end{aligned}
$$

where for compact notation we have set

$$
\llbracket p_{h}^{n+1} \rrbracket^{\prime}= \begin{cases}\llbracket p_{h}^{n+1} \rrbracket & \text { if } F \in \mathcal{F}_{h}^{i *}, \\ \llbracket p_{h}^{n+1} \rrbracket-G\left(s_{h}^{n}\right) & \text { if } F \in \mathcal{F}_{h}^{\Gamma}, \\ p_{h}^{n+1}-p_{D} & \text { if } F \in \mathcal{F}_{h p}^{\mathrm{D}} .\end{cases}
$$

Then, it is readily seen that the first term in (27) weakly enforces the volumetric PDE residual, the second one the flux continuity, the third one the Neumann boundary condition, and the last one the pressure continuity inside $\Omega_{1} \cup \Omega_{2}$, the interface condition on $\Gamma$, and the Dirichlet boundary condition on $\partial \Omega_{p}^{\mathrm{D}}$. Notice in particular that the interface condition enforced weakly is

$$
\llbracket p_{h}^{n+1} \rrbracket=G\left(s_{h}^{n}\right),
$$

which is an $O\left(\tau^{n}\right)$-approximation of $(12)-(13)$.

\subsubsection{Step 2: total velocity reconstruction}

Once the approximate pressure $p_{h}^{n+1}$ has been computed, the total velocity $u_{h}^{n+1}$ is reconstructed in the Raviart-Thomas(-Nédélec) finite element space of degree $l, l \in\{k-1, k\}$, where

$$
\mathbf{R T}_{l}\left(\mathcal{T}_{h}\right):=\left\{u_{h} \in \mathbf{H}(\text { div }) ; \forall T \in \mathcal{T}_{h},\left.u_{h}\right|_{T} \in\left[\mathbb{P}_{l}(T)\right]^{d}+x \mathbb{P}_{l}(T)\right\},
$$



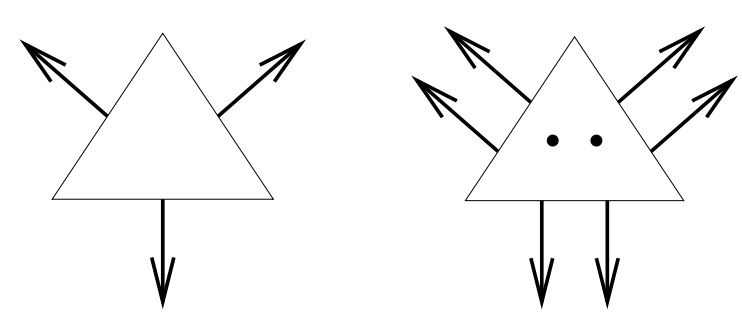

Figure 2: Local degrees of freedom for the spaces $\mathbf{R} \mathbf{T}_{0}$ and $\mathbf{R} \mathbf{T}_{1}$.

where $\mathbf{H}(\operatorname{div})=\left\{v \in\left[L^{2}(\Omega)\right]^{d} ; \nabla \cdot v \in L^{2}(\Omega)\right\}$ and $x=\left(x_{1}, \ldots, x_{d}\right)^{t}$. The reconstruction is presented in the case of matching (that is, without hanging nodes), simplicial meshes. In the more general case, the reconstruction is performed on a matching, simplicial refinement of the original mesh $\mathcal{T}_{h}$.

The reconstruction prescribes locally on each mesh element $T \in \mathcal{T}_{h}$ the local degrees of freedom of $u_{h}^{n+1}$ as follows: For all $F \in \mathcal{F}_{h}$ with $F \subset \partial T$ and for all $q \in \mathbb{P}_{l}(F)$,

$$
\int_{F}\left(u_{h}^{n+1} \cdot n_{F}\right) q=\int_{F}\left(-n_{F} \cdot\left\{\lambda\left(s_{h}^{n}\right) K \nabla p_{h}^{n+1}\right\}_{\omega}+\gamma_{F} \frac{\sigma_{F}}{h_{F}} \llbracket p_{h}^{n+1} \rrbracket^{\prime}\right) q,
$$

except on Neumann boundary faces where there is no jump contribution, and for all $r \in\left[\mathbb{P}_{l-1}(T)\right]^{d}$ (there is no condition if $k=1$ and $l=0$ ),

$$
\int_{T} u_{h}^{n+1} \cdot r=-\int_{T} \lambda\left(s_{h}^{n}\right) K \nabla p_{h}^{n+1} \cdot r+\theta \sum_{F \subset \partial T} \int_{F} \omega_{T, F} \lambda\left(s_{h}^{n}\right) n_{F} \cdot K \cdot r \llbracket p_{h}^{n+1} \rrbracket^{\prime} .
$$

These conditions fully prescribe the degrees of freedom of the vector field $u_{h}^{n+1}$ [9]. The local degrees of freedom for the spaces $\mathbf{R} \mathbf{T}_{0}$ and $\mathbf{R T}_{1}$ are illustrated in Figure 2. Furthermore, the computation of $u_{h}^{n+1}$ is explicit, and thus extremely cheap. Moreover, it is readily seen that the divergence of $u_{h}^{n+1}$ is optimally convergent in the sense that for all $\xi \in \mathbb{P}_{l}(T)$,

$$
\int_{T}\left(\nabla \cdot u_{h}^{n+1}\right) \xi=\int_{T}\left(q_{w}+q_{n}\right) \xi
$$

We refer to [21] for more details.

\subsubsection{Step 3: saturation equation}

To compute $s_{h}^{n+1}$, we use a backward Euler scheme in time, together with a spatial discretization of the diffusion term by an interior penalty DG 
method and a DG method with Godunov flux (or, equivalently, an upwind flux since the fractional flux function is monotone) for the nonlinear advective term. Hence, we solve for $s_{h}^{n+1} \in V_{h}^{k}$ such that for all $v_{h} \in V_{h}^{k}$,

$$
\begin{aligned}
& \sum_{T \in \mathcal{T}_{h}} \int_{T} \phi \tau_{n}^{-1} s_{h}^{n+1} v_{h}-\sum_{T \in \mathcal{T}_{h}} \int_{T} u_{h}^{n+1} f\left(s_{h}^{n+1}\right) \cdot \nabla v_{h}+\sum_{F \in \mathcal{F}_{h}} \int_{F}\left(u_{h}^{n+1} \cdot n_{F}\right) \Phi_{h F}^{n+1} \llbracket v_{h} \rrbracket \\
& +\sum_{T \in \mathcal{T}_{h}} \int_{T} \epsilon\left(s_{h}^{n}\right) \pi^{\prime}\left(s_{h}^{n}\right) \nabla s_{h}^{n+1} \cdot \nabla v_{h} \\
& -\sum_{F \in \mathcal{F}_{h}^{i} \cup \mathcal{F}_{h s}^{\mathrm{D}}}\left(n_{F} \cdot\left\{\epsilon\left(s_{h}^{n}\right) \pi^{\prime}\left(s_{h}^{n}\right) \nabla s_{h}^{n+1}\right\}_{\omega} \llbracket v_{h} \rrbracket+\theta n_{F} \cdot\left\{\epsilon\left(s_{h}^{n}\right) \pi^{\prime}\left(s_{h}^{n}\right) \nabla v_{h}\right\}_{\omega} \llbracket s_{h}^{n+1} \rrbracket\right) \\
& +\sum_{F \in \mathcal{F}_{h}^{i} \cup \mathcal{F}_{h s}^{\mathrm{D}}} \delta_{F} \frac{\sigma_{F}}{h_{F}} \llbracket s_{h}^{n+1} \rrbracket \llbracket v_{h} \rrbracket=\sum_{T \in \mathcal{T}_{h}} \int_{T} \phi \tau_{n}^{-1} s_{h}^{n} v_{h}+\sum_{T \in \mathcal{T}_{h}} \int_{T} q_{n} v_{h} \\
& +\sum_{F \in \mathcal{F}_{h}^{\Gamma}} \int_{F}\left(-\theta n_{F} \cdot\left\{\epsilon\left(s_{h}^{n}\right) \pi^{\prime}\left(s_{h}^{n}\right) \nabla v_{h}\right\}_{\omega}+\delta_{F} \frac{\sigma_{F}}{h_{F}} \llbracket v_{h} \rrbracket\right) J\left(s_{h 1}^{n}\right) \\
& +\sum_{F \in \mathcal{F}_{h s}^{\mathrm{D}}} \int_{F}\left(-\theta n_{F} \cdot \epsilon\left(s_{h}^{n}\right) \pi^{\prime}\left(s_{h}^{n}\right) \nabla v_{h}+\delta_{F} \frac{\sigma_{F}}{h_{F}} v_{h}\right) s_{D},
\end{aligned}
$$

where $s_{D}$ is the Dirichlet datum for the saturation. Although many terms have been linearized by evaluating them at $s_{h}^{n},(34)$ is still a nonlinear problem because of the fractional flux being evaluated at $s_{h}^{n+1}$.

The penalty coefficient $\delta_{F}$ and the weights $\omega$ in the consistency terms are evaluated as described in $\S 3.2$. For $F \in \mathcal{F}_{h}^{i}, F=\partial T^{-} \cap \partial T^{+}$, we set $a_{T^{ \pm}, F}=\left\|\left.\left(\epsilon\left(s_{h}^{n}\right) \pi^{\prime}\left(s_{h}^{n}\right)\right)\right|_{T^{ \pm}}\right\|_{L^{\infty}(F)}$ and for $F \in \mathcal{F}_{h}^{\partial}, F=\partial T \cap \partial \Omega$, we set $a=\left\|\left.\left(\epsilon\left(s_{h}^{n}\right) \pi^{\prime}\left(s_{h}^{n}\right)\right)\right|_{T}\right\|_{L^{\infty}(F)}$. Then, for $F \in \mathcal{F}_{h}^{i *} \cup \mathcal{F}_{h s}^{\mathrm{D}}$, we set $\gamma_{F}=\langle a\rangle_{F}$, and the reference value for $a$ is taken to be $a_{\beta}^{*}:=\max _{s \in\left[S_{n r}, 1-S_{w r}\right)}\left(\epsilon_{\beta}(s) \pi_{\beta}^{\prime}(s)\right)$ where $\beta \in\{1,2\}$ is such that $F \subset \Omega_{\beta}$. For $F \in \mathcal{F}_{h}^{\Gamma}$, the above penalty strategy is revised by setting the weights to $\frac{1}{2}$ and $\delta_{F}=a_{1}^{*} a_{2}^{*} /\left(a_{1}^{*}+a_{2}^{*}\right)$. This way, the interface condition is weakly enforced even in the degenerate case.

The numerical flux $\Phi_{h F}^{n+1}$ is evaluated as

$$
\Phi_{h F}^{n+1}= \begin{cases}f\left(s_{h}^{n+1, \uparrow}\right) & \text { if } F \in \mathcal{F}_{h}^{i *}, \\ \left\{f\left(s_{h}^{n+1}\right)\right\}_{\omega} & \text { if } F \in \mathcal{F}_{h}^{\Gamma}, \\ f\left(s_{h}^{n+1}\right) & \text { if } F \in \mathcal{F}_{h}^{\partial} \backslash \mathcal{F}_{h s}^{\mathrm{D}}, \\ f\left(s_{D}\right) & \text { if } F \in \mathcal{F}_{h s}^{\mathrm{D}},\end{cases}
$$


where $s_{h}^{n+1, \uparrow}$ denotes the so-called upwind value of $s_{h}^{n+1}$, that is, for $F \in \mathcal{F}_{h}^{i}$, $F=\partial T^{-} \cap \partial T^{+}, s_{h}^{n+1, \uparrow}=\left.s_{h}^{n+1}\right|_{T^{-}}$if $u_{h}^{n+1} \cdot n_{F} \geq 0$ and $s_{h}^{n+1, \uparrow}=\left.s_{h}^{n+1}\right|_{T^{+}}$otherwise. The upwind flux is used on all faces except on those at the interface where a weighted centered flux is used. An upwind flux could have been used as well at the interface, but this is unnecessary since the design of the penalty coefficient $\delta_{F}$ already ensures that the interface condition is weakly penalized.

It is interesting to recast (34) in a weak residual form by integrating by parts the second and fourth terms, yielding

$$
\begin{aligned}
& \sum_{T \in \mathcal{T}_{h}} \int_{T}\left(\phi \tau_{n}^{-1}\left(s_{h}^{n+1}-s_{h}^{n}\right)+\nabla \cdot\left(u_{h}^{n+1} f\left(s_{h}^{n+1}\right)-\epsilon\left(s_{h}^{n}\right) \pi^{\prime}\left(s_{h}^{n}\right) \nabla s_{h}^{n+1}\right)-q_{n}\right) v_{h} \\
& +\sum_{F \in \mathcal{F}_{h}^{i}} \int_{F} n_{F} \cdot \llbracket-u_{h}^{n+1} f\left(s_{h}^{n+1}\right)+\epsilon\left(s_{h}^{n}\right) \pi^{\prime}\left(s_{h}^{n}\right) \nabla s_{h}^{n+1} \rrbracket\left\{v_{h}\right\}_{\bar{\omega}} \\
& +\sum_{F \in \mathcal{F}_{h}^{\partial} \backslash \mathcal{F}_{h s}^{\mathrm{D}}} \int_{F} n_{F} \cdot \epsilon\left(s_{h}^{n}\right) \pi^{\prime}\left(s_{h}^{n}\right) \nabla s_{h}^{n+1} v_{h} \\
& +\sum_{F \in \mathcal{F}_{h}^{i} \cup \mathcal{F}_{h s}^{\mathrm{D}}} \int_{F}\left(-\theta n_{F} \cdot\left\{\epsilon\left(s_{h}^{n}\right) \pi^{\prime}\left(s_{h}^{n}\right) \nabla v_{h}\right\}_{\omega}+\delta_{F} \frac{\sigma_{F}}{h_{F}} \llbracket v_{h} \rrbracket\right) \llbracket s_{h}^{n+1} \rrbracket^{\prime} \\
& +\sum_{F \in \mathcal{F}_{h}^{i *}} \int_{F} \omega_{T \downarrow}\left|u_{h}^{n+1} \cdot n_{F}\right| \llbracket v_{h} \rrbracket \llbracket f\left(s_{h}^{n+1}\right) \rrbracket+\sum_{F \in \mathcal{F}_{h s}^{\mathrm{D}}} \int_{F}\left|u_{h}^{n+1} \cdot n_{F}\right| \llbracket v_{h} \rrbracket \llbracket s_{h}^{n+1} \rrbracket^{\prime}=0,
\end{aligned}
$$

where for compact notation we have set

$$
\llbracket s_{h}^{n+1} \rrbracket^{\prime}= \begin{cases}\llbracket s_{h}^{n+1} \rrbracket & \text { if } F \in \mathcal{F}_{h}^{i *}, \\ \llbracket s_{h}^{n+1} \rrbracket-J\left(s_{h 1}^{n}\right) & \text { if } F \in \mathcal{F}_{h}^{\Gamma}, \\ s_{h}^{n+1}-s_{D} & \text { if } F \in \mathcal{F}_{h s}^{\mathrm{D}} .\end{cases}
$$

Thus, the first term in (36) weakly enforces the volumetric PDE residual, the second one the flux continuity, the third one the Neumann boundary condition, and the fourth one the saturation continuity inside $\Omega_{1} \cup \Omega_{2}$, the interface condition on $\Gamma$, and the Dirichlet boundary condition on $\partial \Omega_{s}^{\mathrm{D}}$. We now clearly see why the penalty coefficient $\delta_{F}$ must be nonzero at the interface even in the degenerate case. Furthermore, the interface condition enforced weakly is

$$
\llbracket s_{h}^{n+1} \rrbracket=J\left(s_{h 1}^{n}\right)
$$


which is an $O\left(\tau^{n}\right)$-approximation of (10). Finally, the two terms in the fifth line of (36) result from upwinding. In the first of the two terms, $T^{\downarrow}$ denotes the mesh element downwind of $F$; moreover, this term is dissipative since $f$ is an increasing function (that is, it yields a nonnegative contribution upon taking $v_{h}=s_{h}^{n+1}$ ). In the second term, we have used the fact that $\partial \Omega_{s}^{\mathrm{D}}$ coincides with the inflow boundary to introduce the absolute value of $u_{h}^{n+1} \cdot n_{F}$; moreover, this second term is also dissipative.

\section{Numerical results}

The DG method designed in $\S 3$ is assessed on three one-dimensional test cases with synthetic data representative of secondary oil recovery problems exhibiting degeneracies (the non-wetting phase saturation vanishes in some parts of the domain) together with pressure and saturation discontinuities at the interface between the two subdomains. The computational domain is $\Omega=(0,2)$ with the interface located at $x_{i n t f}=1$ so that $\Omega_{1}=(0,1)$ and $\Omega_{2}=(1,2)$. In both subdomains, we set $\phi=0.2, S_{n r}=S_{w r}=0$, and choose the mobilities from Brooks-Corey model [6] with parameter $\theta=2$, yielding for $s \in[0,1]$,

$$
\lambda_{w}(s)=(1-s)^{4}, \quad \lambda_{n}(s)=s^{2}\left(2 s-s^{2}\right) .
$$

The absolute permeability is specified below for each test case. The capillary pressures are given for $s \in[0,1]$ by

$$
\pi_{1}(s)=5 s^{2}, \quad \pi_{2}(s)=4 s^{2}+1,
$$

so that the difference in entry pressures between the two subdomains is equal to 1 . The critical saturation is $S^{*}=\frac{1}{\sqrt{5}} \simeq 0.4472$. There are no sources or sinks $\left(q_{w}=q_{n}=0\right)$. We enforce Dirichlet boundary conditions on the pressure in the form $\left.p\right|_{x=0}=1.8$ and $\left.p\right|_{x=2}=1.0$, and mixed boundary conditions on the saturation in the form $\left.s\right|_{x=0}=0$ and $\left.\epsilon(s) \pi^{\prime}(s) \frac{d s}{d x}\right|_{x=2}=0$. The initial condition is specified below for each test case.

We use a uniform mesh of $\Omega$ composed of 80 elements in each subdomain, together with first-order polynomial approximation for both the pressure and the saturation $(k=1$ in $(22))$, and the total velocity is reconstructed in the Raviart-Thomas(-Nédélec) finite element space $\mathbf{R T}_{1}(l=1$ in $§ 3.3 .2)$. The time step is set to 0.001. We have verified that the adopted mesh size and time step yield sufficient accuracy. Furthermore, since we are approximating rough 
solutions with possible degeneracies, spurious oscillations in the saturation profiles triggered by Gibbs-type phenomena can and do occur. As reflected in the results below, these oscillations always remain localized in space to a few cells around shocks, and their amplitude is always very small. Thus, simulations can be performed without employing limiters. To this purpose, the mobilities and capillary pressures are extended by constant values outside the interval $[0,1]$.

In test case 1 , the intrinsic permeability is set to $K=1$ over the whole domain. The initial condition for the saturation is

$$
s(x, t=0)= \begin{cases}0.9 & \text { if } x \in(0.1,0.9) \\ 0 & \text { otherwise }\end{cases}
$$

so that the non-wetting phase is initially present only in the left subdomain. Results are presented in Figure 3. The saturation profiles are typical of propagating fronts with degeneracies and capillary pressure effects. Some spurious oscillations are visible in the few cells around the propagating fronts at time $t=0.015$; these oscillations always remain localized in space and are damped out already at time $t=0.045$. When the saturation reaches the critical value $S^{*}$ at the interface, the non-wetting phase penetrates into the right subdomain, and the saturation remains discontinuous at the interface. Regarding the pressure profiles, the total pressure is discontinuous at the interface, the capillary pressure is first discontinuous and becomes continuous once the non-wetting phase has penetrated inside the right subdomain, in agreement with the interface conditions, and the wetting-phase pressure is continuous before and after penetration, again in agreement with the interface conditions. In addition, it is interesting to observe that owing to the total pressure jump at the interface, the total pressure gradient in each subdomain is larger than the gradient that could be expected simply from the values of the Dirichlet boundary conditions for the pressure.

Figure 4 compares the above results, for which the total velocity is accurately reconstructed, to those obtained by simply using the piecewise constant pressure gradient to compute the total velocity. It is seen that this latter procedure leads to substantial spurious oscillations and even to the formation of non-physical shocks at times $t=0.045$ and $t=0.1$. By comparing the left and right columns of Figure 4, it is observed that these oscillations are localized where the total velocity is not accurately reconstructed. Finally, at longer simulation times (e.g., $t=0.25$ ) when the saturation profiles 
have become smoother, these spurious oscillations are damped out. These results clearly demonstrate the need for accurate velocity reconstruction in two-phase flow simulations.

Test case 2 extends test case 1 to the situation where the intrinsic permeability is heterogeneous, namely $K_{1}=1$ and $K_{2}=0.1$. The same initial condition is used as in test case 1. Results are presented in Figure 5. As for test case 1 , the non-wetting phase can penetrate into the subdomain $\Omega_{2}$, but because $K_{2}$ is now smaller, the non-wetting phase propagates more slowly to the right in $\Omega_{2}$, thereby also inducing a different saturation profile in $\Omega_{1}$ (compare the result at $t=0.25$ with that of Figure 3 ). The saturation exhibits a jump at the interface, and a few spurious oscillations are present in the few cells before the propagating front. The total pressure is discontinuous at the interface, the capillary pressure is first discontinuous and then continuous, while the wetting-phase pressure is always continuous, in agreement with the enforced interface conditions.

Test case 3 uses the same data as test case 2 except that the initial value of the saturation for $x \in(0.1,0.9)$ is now 0.4 . Thus, when the propagating front reaches the interface, the saturation is below the critical value so that the non-wetting phase cannot immediately penetrate inside $\Omega_{2}$. The nonwetting phase then accumulates upstream of the interface until it reaches eventually the critical value $S^{*}$ and penetrates inside $\Omega_{2}$; this situation is typical of oil trapping effects. Furthermore, the pressure profiles are again in agreement with the the enforced interface conditions.

To conclude, the above results illustrate that the proposed DG method is capable of tackling in a robust and accurate fashion various physical phenomena relevant to two-phase flow problems in heterogeneous porous media with discontinuous capillary pressures. The nonlinear interface conditions, which are weakly enforced in the context of a sequential scheme, are well captured in the numerical simulations. The total velocity reconstruction and the weighted averages are important ingredients to achieve stable computations that can be carried over without using limiters even in degenerate cases. Our aim is now to assess more thoroughly the proposed method in the context of multi-dimensional heterogeneous problems in more complex and realistic test cases related to field studies. 


\section{References}

[1] B. Amaziane, A. Bourgeat, and H. El Amri. Existence of solutions to various rock types model of two-phase flow in porous media. Appl. Anal., 60(1-2):121-132, 1996.

[2] K. Aziz and A. Sttari. Petroleum Resevoir Simulation. Applied Science Publishers Ltd., 1979.

[3] F. Bassi, S. Rebay, G. Mariotti, S. Pedinotti, and M. Savini. A highorder accurate discontinuous finite element method for inviscid and viscous turbomachinery flows. In R. Decuypere and G. Dibelius, editors, Proceedings of the $2^{\text {nd }}$ European Conference on Turbomachinery Fluid Dynamics and Thermodynamics, pages 99-109, 1997.

[4] P. Bastian and B. Rivière. Superconvergence and H(div) projection for discontinuous Galerkin methods. Internat. J. Numer. Methods Fluids., 42(10):1043-1057, 2003.

[5] P. Bastian and B. Rivière. Discontinuous Galerkin methods for twophase flow in porous media. Technical Report 2004-28, 2004.

[6] J. Bear. Dynamic of Fluids in Porous Media. Dover, New York, New York, 1978.

[7] M. Bertsch, R. Dal Passo, and C. J. Van Duijn. Analysis of oil trapping in porous media flow. SIAM J. Math. Anal., 35:245-267, 2003.

[8] A. Bourgeat and A. Hidani. Effective model of two-phase flow in a porous medium made of different rock types. Appl. Anal., 58(1-2):1-29, 1995.

[9] F. Brezzi and M. Fortin. Mixed and Hybrid Finite Element Methods, volume 15 of Springer Series in Computational Mathematics. SpringerVerlag, New York, 1991.

[10] C. Cancès. Nonlinear parabolic equations with spatial discontinuities. NoDEA, 15:427-456, 2008.

[11] C. Cancès. Finite volume scheme for two-phase flow in heterogeneous porous media involving capillary pressure discontinuities. Technical report, arXiv:0902.1873v1, 2009. 
[12] C. Cancès, T. Gallouët, and A. Porretta. Two-phase flows involving capillary barriers in heterogeneous porous media. Interfaces and Free Boundaries, To appear, 2009.

[13] G. Chavent and J. Jaffré. Mathematical Models and Finite Elements for Reservoir Simulation. Elsevier, North-Holland, 1978.

[14] Z. Chen, R. E. Ewing, and M. Espedal. Multiphase flow simulation with various boundary conditions. In Proceedings of the International Conference on Computational Methods in Water Resources X, pages 925-932, 1994.

[15] Z. Chen, G. Huan, and Y. Ma. Computational Methods for Multiphase Flows in Porous Media. Siam, 2006.

[16] D. A. Di Pietro, A. Ern, and J.-L. Guermond. Discontinuous Galerkin methods for anisotropic semidefinite diffusion with advection. SIAM J. Numer. Anal., 46(2):805-831, 2008.

[17] G. Enchéry, R. Eymard, and A. Michel. Numerical approximation of a two-phase flow problem in a porous medium with discontinuous capillary forces. SIAM J. Numer. Anal., 43(6):2402-2422, 2006.

[18] Y. Epshteyn and B. Rivière. Fully implicit discontinuous finite element methods for two-phase flow. Applied Numerical Mathematics, 57:383401, 2007.

[19] A. Ern and J.-L. Guermond. Discontinuous Galerkin methods for Friedrichs' systems. I. General theory. SIAM J. Numer. Anal., 44(2):753-778, 2006.

[20] A. Ern, I. Mozolevski, and L. Schuh. Accurate velocity reconstruction for Discontinuous Galerkin approximations of two-phase porous media flows. C. R. Acad. Sci. Paris, Ser. I, To appear, 2009.

[21] A. Ern, S. Nicaise, and M. Vohralík. An accurate H(div) flux reconstruction for discontinuous Galerkin approximations of elliptic problems. $C$. R. Math. Acad. Sci. Paris, 345(12):709-712, 2007.

[22] A. Ern, A. F. Stephansen, and P. Zunino. A discontinuous Galerkin method with weighted averages for advection-diffusion equations with 
locally small and anisotropic diffusivity. IMA J. Numer. Anal., To appear, 2009.

[23] B. G. Ersland, M. S. Espedal, and R. Nybø. Numerical methods for flow in a porous medium with internal boundaries. Comput. Geosci., $2(3): 217-240,1998$.

[24] O. J. Eslinger. Discontinuous Galerkin Finite Element Methods apllied to two-phase, air-water flow problems. PhD thesis, University of Texas at Austin, 2005.

[25] R. Helmig. Multiphase Flow and Transport Processes in the Subsurface. Springer, 1997.

[26] W. Klieber and B. Rivière. Adaptive simulations of two-phase flow by discontinuous Galerkin methods. Comput. Methods Appl. Mech. Engrg., 196(1-3):404-419, 2006.

[27] C. J. Van Duijn, J. Molenaar, and M. J. Neef. The effect of capillary forces on immiscible two-phase flow in heterogeneous porous media. Transport in Porous Media, 21:71-93, 1995. 

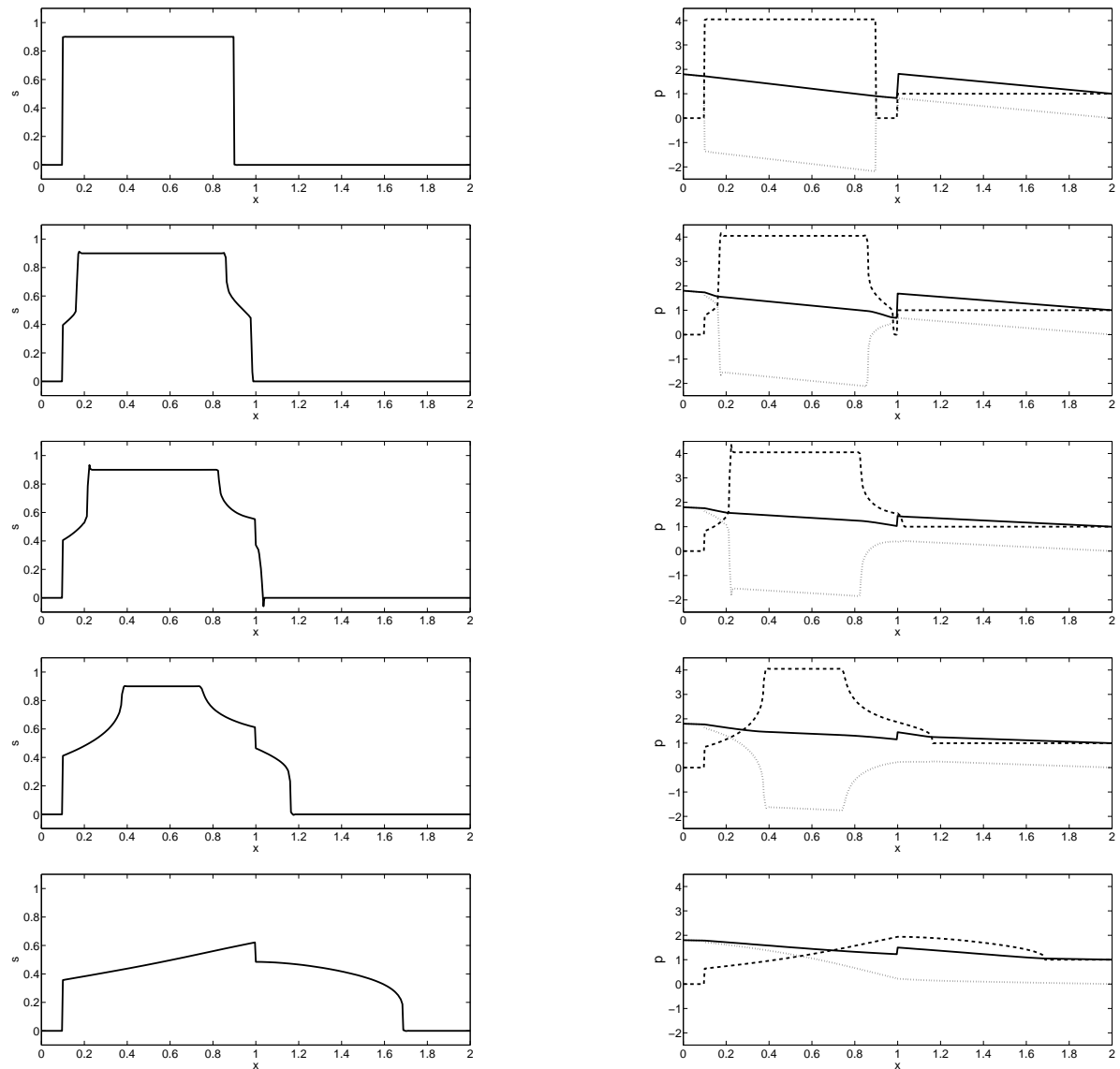

Figure 3: Test case 1. Left: saturation profiles at various times; right: total pressure (solid), capillary pressure (dashed), and wetting-phase pressure (dotted) at different times. From top to bottom: $t=0, t=0.008, t=0.015, t=0.045$, and $t=0.25$. 

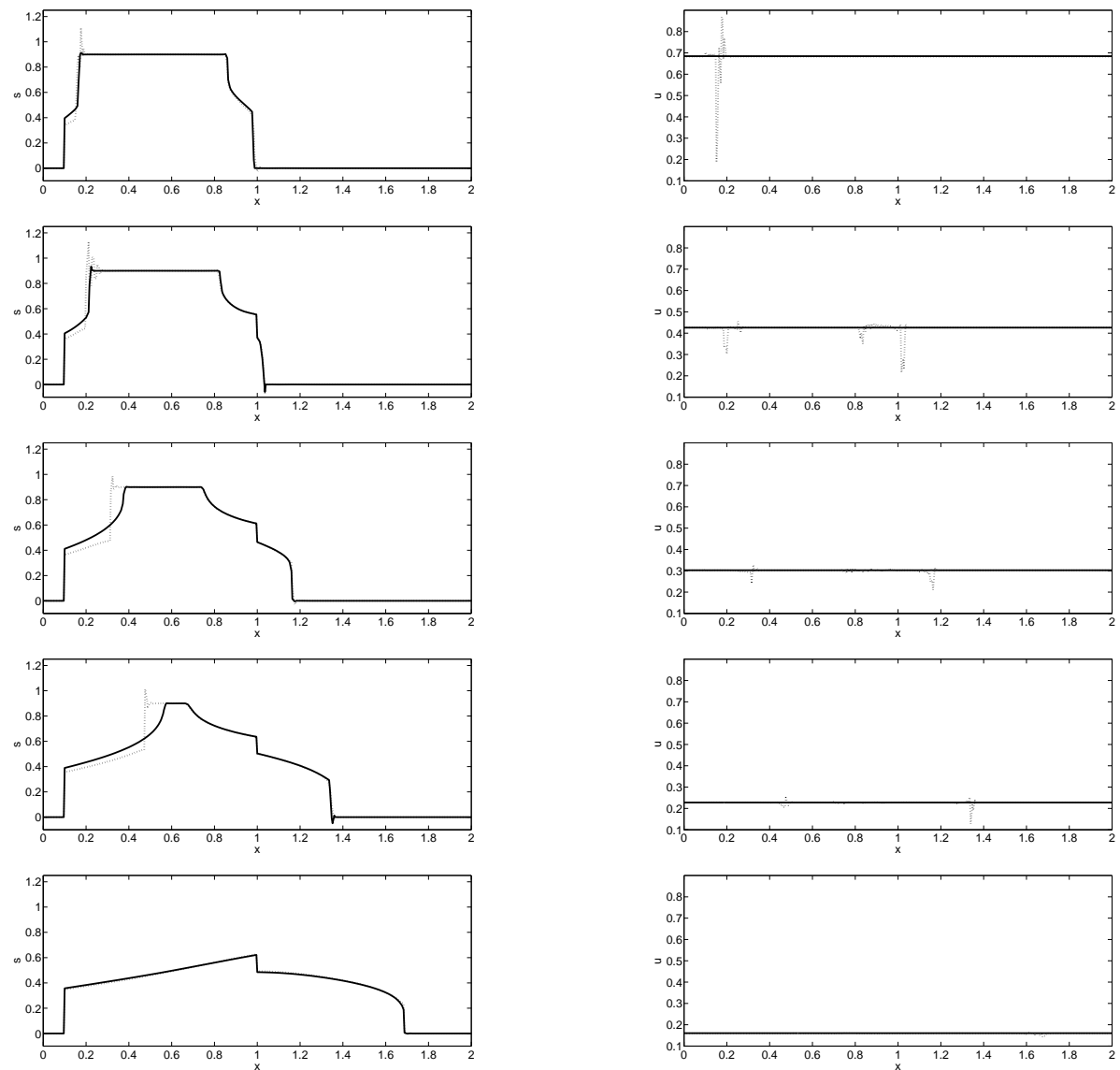

Figure 4: Test case 1. Comparison of results obtained with (solid lines) and without (dashed lines) velocity reconstruction: saturation (left) and total velocity (right) at different times. From top to bottom: $t=0.008, t=0.015, t=0.045, t=0.1$, and $t=0.25$. 

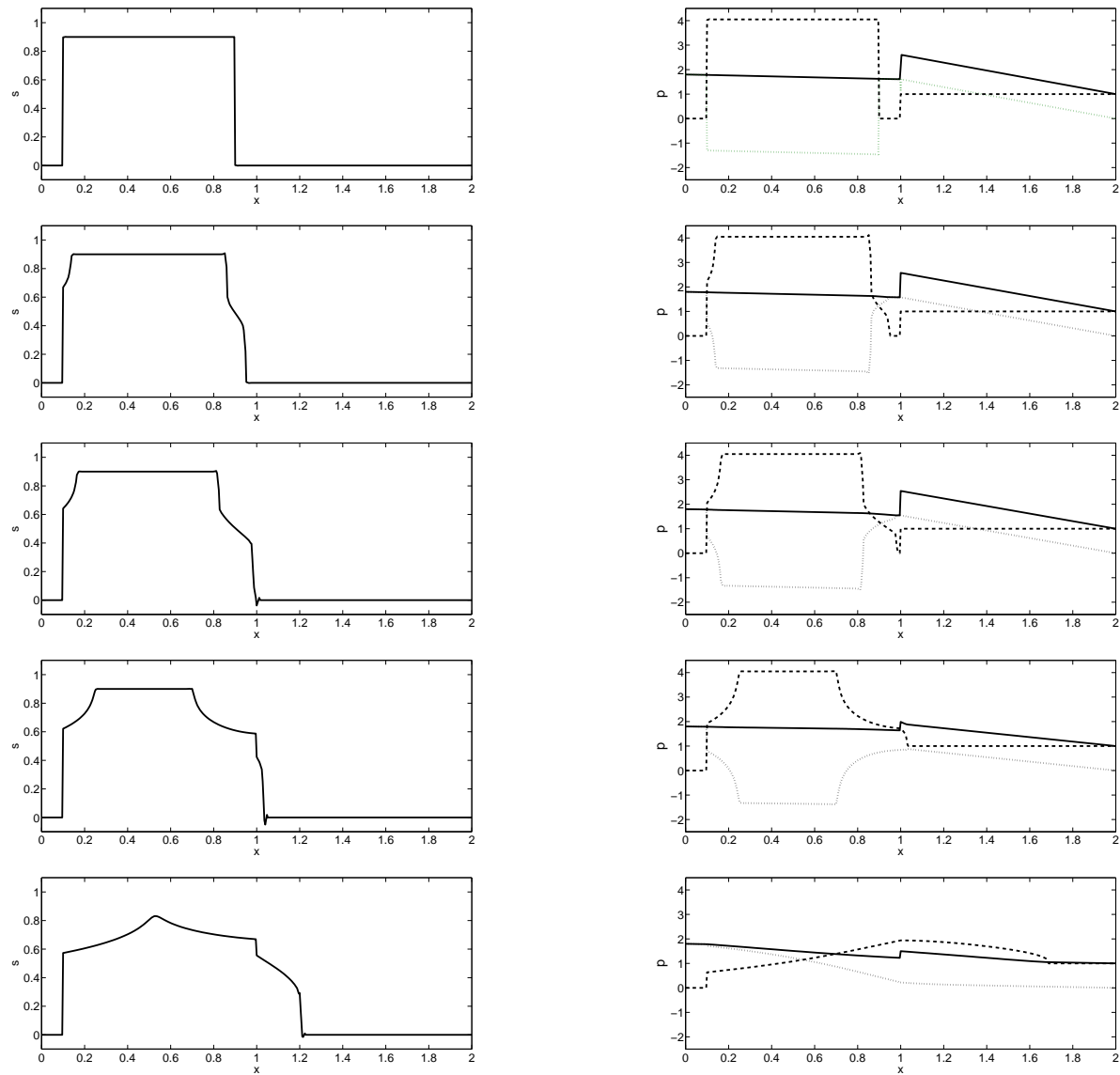

Figure 5: Test case 2. Left: saturation profiles at various times; right: total pressure (solid), capillary pressure (dashed), and wetting-phase pressure (dotted) at different times. From top to bottom: $t=0, t=0.015, t=0.018, t=0.03$, and $t=0.25$. 

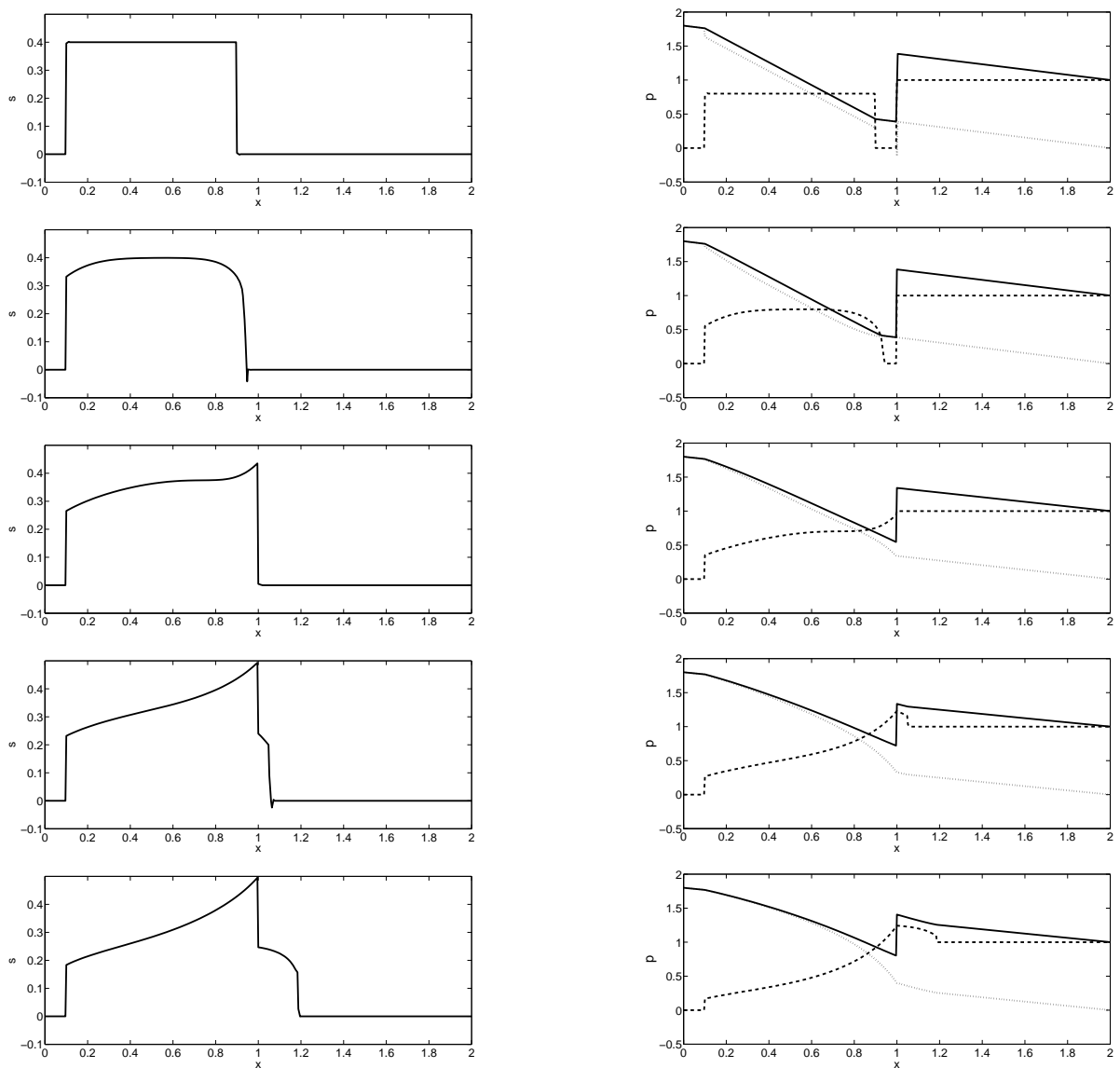

Figure 6: Test case 3. Left: saturation profiles at various times; right: total pressure (solid), capillary pressure (dashed), and wetting-phase pressure (dotted) at different times. From top to bottom: $t=0, t=0.008, t=0.045, t=0.1$, and $t=0.25$. 